\title{
Ausgewählte Fragen im Zusammenhang mit dem Gewebegesetz
}

\author{
Horst Hasskarl Bita Bakhschai \\ Kanzlei Harms Hasskarl Melzer, Ludwigshafen, Deutschland
}

\section{Schlüsselwörte}

Gewerbezubereitungen - Herstellung und Anwendung durch Arzt · Herstellungserlaubnis · Vertriebsgenehmigung

\section{Zusammenfassung}

Auch nach Inkrafttreten des Gewebegesetzes ist die erlaubnisfreie ärztliche Eigenherstellung einer Gewebezubereitung im Sinne des $\S 4$ Abs. 30 Arzneimittelgesetz (AMG) zur Anwendung am eigenen Patienten nach § 4a Satz $1 \mathrm{Nr}$. 3 AMG unter der unmittelbaren fachlichen Verantwortung des herstellenden und anwendenden Arztes unverändert zulässig. Auch besteht unverändert die Möglichkeit, dabei weisungsgebundene Hilfspersonen einzuschalten. Das AMG findet in diesem Fall insgesamt keine Anwendung. Liegen die Voraussetzungen einer ärztlichen Eigenherstellung nach § 4a Satz $1 \mathrm{Nr}$. 3 AMG nicht vor, ist die Gewinnung des Gewebes nach § 20b AMG und die Be- oder Verarbeitung, Konservierung, Lagerung oder das Inverkehrbringen von Gewebe oder Gewebezubereitungen nach § 20c AMG erlaubnispflichtig. Eine Genehmigung zum Inverkehrbringen von Gewebezubereitungen nach § 21a AMG ist wegen des fehlenden zulassungsrechtlich relevanten Inverkehrbringens im Sinne des § 4 Abs. 17 AMG jedenfalls dann nicht erforderlich, wenn der Leiter einer Klinikabteilung unmittelbar für die Herstellung der Gewebezubereitung in seiner Abteilung zuständig ist und sie auch in seiner Abteilung anwendet, wenn auch unter Zuhilfenahme seiner ihm unterstellten Ärzte. Nach der weitergehenden Auffassung der Autoren gilt dies auch dann, wenn die in einem Krankenhaus hergestellte Gewebezubereitung innerhalb desselben Krankenhausträgers (derselben öffentlich-rechtlichen oder privatrechtlichen juristischen Person) an einen anderen als den herstellenden Arzt zur Anwendung an seinem Patienten abgegeben wird, sofern durch die innerbetriebliche Organisation der unmittelbare rechtliche Einwirkungsbereich des Krankenhausträgers fortbesteht. Es liegt dann keine "Abgabe an andere" und damit kein Inverkehrbringen im Sinne des § 21a Abs. 1 Satz 1 AMG vor.

\author{
Key Words \\ Tissue preparations - Manufacture and administration \\ by physician - Manufacturing authorization - Marketing \\ authorization
}

\section{Summary}

Selected Questions Relating to the Tissue Law

After the coming into force of the tissue law the manufacture by a physician of a medicinal product as tissue preparation in the meaning of section 4 subp. 30 German drug law (AMG) without manufacturing authorization issued by the competent authority remains permissible as before, if the medicinal product is administered to the physician's patients pursuant to section $4 \mathrm{a}$ sentence 1 No. 3 AMG and if the direct professional responsibility for the manufacture and the administration of the medicinal product remains with physician. The physician has the possibility to use the services of auxiliary personnel provided, however, this personnel acts only on the basis of instructions given by the physician. In such a case the AMG as a whole is not applicable. If, however, the prerequisites of the manufacture of a medicinal product by a physician pursuant to section 4a sentence 1 No. 3 AMG are not met, the production of human tissue pursuant to section 20b AMG and the processing, preservation, storage or distribution of tissue and tissue preparations pursuant to section 20c AMG are dependent on a manufacturing authorization. A marketing authorization for preparations in accordance with section 21 a AMG is not required because of the non-fulfillment of the definition 'placing on the market' in the meaning of section 4 subp. 17 AMG. In any case this applies if the head of a clinical department is directly responsible for the manufacture of the tissue preparation in his department and if the tissue preparation is administered in his department, even though he uses the services of physicians working under him. It is the opinion of the authors that this legal situation remains unchanged, even if the tissue preparation manufactured in a hospital by one physician will be supplied to another physician in the same hospital not having manufactured the medicinal product and which physician administers the product to his patients. As long as the owner of the hospital, be it a public-law or a private-law legal entity, remains the same and under the condition that - as a result of the internal organization - the direct legal influence and competence of the owner of the hospital continues to exist, no handing over takes place in a legal sense. In that case the definition 'distribution to others' and therefore the placing on the market within the meaning of section 21 a subp. 1 sentence 1 AMG is not fulfilled.

\begin{tabular}{ll}
\hline KARGER & @ 2008 S. Karger GmbH, Freiburg \\
Fax +497614520714 & Accessible online at: \\
Information@Karger.de & www.karger.com/tmh \\
www.karger.com &
\end{tabular}




\section{Einleitung}

Die verdienstvolle Darstellung der Hintergründe und Konsequenzen des Gewebegesetzes durch von Auer [1] lässt Raum für eine weitergehende, vertiefende Darstellung von ausgewählten Fragen, die sich dem Rechtsunterworfenen in diesem Zusammenhang stellen. Nachstehend wird der Versuch unternommen, einzelne Fragestellungen und Problemkreise, die sich im Zusammenhang mit dem Inkrafttreten des Gewebegesetzes [2] und aufgrund der beratenden Tätigkeit der Autoren ergeben haben, darzustellen.

So hat sich z.B. in der Praxis gezeigt, dass zum Teil Unklarheiten über die Möglichkeiten der ärztlichen Eigenherstellung einer Gewebezubereitung durch den dieses Arzneimittel an seinem Patienten anwendenden Arzt bestehen. Hier stellt sich die Frage, ob und unter welchen Voraussetzungen dies nach § 4a Satz 1 Nr. 3 AMG [3] weiterhin möglich ist. Das hat gegebenenfalls zur Folge, dass auf diese Herstellungstätigkeit das AMG insgesamt keine Anwendung findet und also eine Herstellungserlaubnis nicht erforderlich ist. Ebenso ist unklar inwieweit bei der Herstellung Hilfspersonen eingeschaltet werden können.

Ferner ist es von erheblichem Interesse, in welchen Fällen für das Inverkehrbringen einer Gewebezubereitung eine Genehmigung nach § 21a AMG erforderlich ist. Dabei konzentriert sich die Darstellung auf die Frage, ob eine «Abgabe an andere» als Unterfall des Inverkehrbringens im Sinne des $\S 4$ Abs. 17 AMG zwingend dann vorliegt, wenn eine in einer Abteilung eines Krankenhauses hergestellte Gewebezubereitung an einen Arzt in einer anderen Abteilung desselben Krankenhauses zur Anwendung an seinem Patienten abgegeben wird.

\section{Ärztliche Eigenherstellung von Gewebezubereitungen nach § 4a Satz 1 Nr. 3 AMG}

Die durch das Gewebegesetz nicht geänderte Vorschrift des 4a Satz 1 Nr. 3 AMG regelt die Voraussetzungen, unter denen die ärztliche Eigenherstellung von Arzneimitteln zur Anwendung am eigenen Patienten möglich ist, ohne dass der Vorgang dem Anwendungsbereich des AMG unterliegt. Die Vorschrift lautet:

«Dieses Gesetz findet keine Anwendung auf ...

3. Arzneimittel, die ein Arzt, Tierarzt oder eine andere Person, die zur Ausübung der Heilkunde befugt ist, bei Mensch oder Tier anwendet, soweit die Arzneimittel ausschließlich zu diesem Zweck unter der unmittelbaren fachlichen Verantwortung eines Arztes, Tierarztes oder der anwendenden Person, die zur Ausübung der Heilkunde befugt ist, hergestellt worden sind, ...»

Maßgebend ist daher, dass der herstellende und zugleich das Arzneimittel anwendende Arzt das Arzneimittel unter seiner unmittelbaren fachlichen Verantwortung herstellt, um es ausschließlich an seinem Patienten anzuwenden.
$\mathrm{Zu}$ klären ist daher, was unter der unmittelbaren fachlichen Verantwortung des anwendenden Arztes zu verstehen ist. Dies ist im Wege der Gesetzesauslegung zu ermitteln.

Nach dem Wortlaut des $\S 4$ a Satz 1 Nr. 3 AMG fordert das Gesetz nicht die Herstellung des Arzneimittels durch den anwendenden Arzt, sondern lediglich, dass die Herstellung unter seiner unmittelbaren fachlichen Verantwortung erfolgt.

Daher ist die Möglichkeit, eine Hilfsperson einzuschalten, gesetzlich keineswegs ausgeschlossen. Es kommt vielmehr und entscheidend darauf an, dass der Arzt seine fachliche Verantwortung jederzeit und unmittelbar wahrnehmen kann. Er allein bleibt für die Herstellung des Arzneimittels rechtlich verantwortlich. Die Hilfspersonen müssen ihm direkt zugeordnet sein, so dass er seine Verantwortung durch die insoweit allein ihm zustehende Weisungsbefugnis direkt gegenüber diesem Personenkreis ohne Weiteres wahrnehmen kann. Zur Ausübung dieses Weisungsrechts ist nach Auffassung der Autoren jedoch nicht die ständige körperliche Präsenz während des Herstellungsvorgangs erforderlich, wenn auch wünschenswert. Ausreichend dürfte insoweit nach Unterweisung der Hilfspersonen, die sporadische körperliche Anwesenheit, die ständige räumliche Nähe und vor allem die ständige Erreichbarkeit des anwendenden - und für die Herstellung verantwortlichen - Arztes sein. Eine solche weisungsgebundene Hilfsperson des herstellenden und anwendenden Arztes könnte z.B. ein Assistenzarzt oder ein (leitender) OP-Pfleger sein. Dabei ist zu betonen, dass allein der anwendende Arzt für die Herstellung des Arzneimittels rechtlich verantwortlich bleibt. Eine Einzelmeinung in der Literatur vertritt hierzu allerdings eine andere Ansicht. Danach wird die persönliche Anwesenheit des Arztes während des Herstellungsvorgangs und die Ausführung durch eine ihm gegenüber weisungsgebundene Person, falls er nicht selbst herstellt, gefordert [4]. Aber auch nach dieser Literaturmeinung besteht die Möglichkeit, Hilfspersonen einzuschalten.

Die Richtigkeit der hier vertretenen Auffassung wird im Übrigen bestätigt durch das Urteil des Bundesverwaltungsgerichts (BVerwG) vom 18. März 2004 [5], in dem es wie folgt heißt:

«...Das Gesetz erfasst hiernach die Herstellung solcher Arzneimittel nicht, die ein Arzt zur Anwendung bei seinen Patienten selbst herstellt bzw. durch ihm unterstelltes Personal herstellen lässt ...»

In differenzierender Weise sind neuestens auch Bundesärztekammer und die Kassenärztliche Bundesvereinigung zu diesem Ergebnis gelangt [23]. Etwas anderes ergibt sich auch nicht bei historischer Auslegung des § 4a Satz 1 Nr. 3 AMG.

Die Einfügung dieser Norm erfolgte bekanntermaßen, um dem Urteil des Bundesverfassungsgerichts (BVerfG) vom 16. Februar 2000, Az.: 1 BvR 420/97, zur Frischzellen-Verordnung Rechnung zu tragen. Das BVerfG hatte sich mit der ärztlichen Therapiefreiheit und dem Inverkehrbringen eines von einem Arzt hergestellten und zur Anwendung an einem Patienten bestimmten Arzneimittels auseinandergesetzt. Aufgrund des 
zu entscheidenden Sachverhalts hatte sich das BVerfG lediglich mit der Frage auseinandergesetzt, inwieweit bei der Anwendung eines Arzneimittels der Einsatz von Hilfskräften zulässig ist, ohne dass eine arzneimittelrechtlich relevante Abgabe an andere vorliegt. Die Beschwerdeführer hatten allesamt das Arzneimittel mit ihren eigenen Händen hergestellt, so dass sich das BVerfG diesbezüglich nicht zu äußern brauchte. Dementsprechend heißt es in der Entscheidung wie folgt:

\begin{abstract}
«Für das Arzneimittelrecht besteht daher in Rechtsprechung und Literatur Einigkeit darüber, dass bei der Herstellung durch einen Arzt, der das von ihm hergestellte Arzneimittel selbst am Patienten anwendet oder in seinen [m] unmittelbaren Einwirkungsbereich durch weisungsgebundene Hilfskräfte oder durch den Patienten selbst anwenden lässt, keine Abgabe in diesem Sinne vorliegt ... Ärzte brauchen deshalb keine Herstellungserlaubnis, solange sie die von ihnen hergestellten Arzneimittel nicht aus der Hand geben. Wenn das Arzneimittel an die Patienten oder an andere Ärzte weitergeben wird und damit die Verfügungsgewalt über das Arzneimittel wechselt, ist hingegen unter den Voraussetzungen des $\S 13$ AMG eine Herstellungserlaubnis erforderlich.» (Hervorhebungen durch die Verfasser)
\end{abstract}

Auch wenn zwischenzeitlich die konkurrierende Gesetzgebungskompetenz nach Artikel 74 Abs. 1 Nr. 19 Grundgesetz (GG) das Recht der Arzneien umfasst und nicht - wie vormals geregelt - nur den Verkehr mit Arzneien, besteht § 4a Satz 1 Nr. 3 AMG unverändert fort.

Insbesondere wurde die Vorschrift auch nicht durch das Gewebegesetz geändert. Eine Änderung hat lediglich § 4a Satz 1 Nr. 4 AMG erfahren, wonach das AMG keine Anwendung findet auf Gewebe, die innerhalb eines Behandlungsvorgangs einer Person entnommen werden, um auf diese rückübertragen zu werden (autologes Transplantat).

Etwas anderes ergibt sich auch nicht aus der Gesetzesbegründung zur ursprünglich geplanten Streichung der Vorschrift des $\S 4$ a Satz 1 Nr. 4 AMG (BR-Drs. 543/06 vom 11. August 2006, S. 85), die wie folgt lautete:

\begin{abstract}
«... Aus Qualitäts- und Sicherheitsgründen werden die in Nr. 4 genannten Gewebe-Tätigkeiten den Anforderungen des Arzneimittelrechts unterworfen, wie sie für den sonstigen Umgang mit Gewebe seit Langem auch gelten. Es bleibt aus Gründen der Therapiefreiheit die Regelung in Nummer 3 bestehen, die nach wie vor die Entnahme und Anwendung von Gewebe durch ein und dieselbe ärztliche Person von den Vorschriften des Arzneimittelrechts ausnimmt. Die Streichung bedeutet insbesondere, dass Entnahme und Verarbeitung, die nicht «in einer Hand» stattfinden, künftig herstellungserlaubnispflichtig sind. Die erforderliche Umstellung der davon betroffenen Gewebeeinrichtungen wird dadurch erleichtert, dass nach $\S 14$ Abs.2b AMG der Leiter der Herstellung zugleich der Leiter der Qualitätskontrolle sein kann und die Entnahmeanforderungen deutlich gegenüber den Anforderung an die Verarbeitung abgesenkt sind ...»
\end{abstract}

Der Gesetzgeber stellt hier klar, dass § 4a Satz 1 Nr. 3 AMG unverändert fortbesteht, wobei er sich nicht näher mit dem Einhandprinzip auseinandersetzt. Schließlich ging es hier um die geplante Streichung einer Ausnahme vom Anwendungsbereich des AMG, nämlich der Nr. 4 für Organe, Organteile und Gewebe, und nicht um die ärztliche Eigenherstellung eines Arzneimittels nach $\S 4$ a Satz 1 Nr. 3 AMG. Im Übrigen wird nicht das entnommene Gewebe am Patienten angewendet, sondern ein Arzneimittel im Sinne des § 2 Abs. 1 AMG, das aus entnommenem Gewebe im Sinne des. § 1a Nr. 4 TPG hergestellt worden und somit eine Gewebezubereitung im Sinne des $\S 4$ Abs. 30 AMG ist. Hätte der Gesetzgeber gewollt, dass die Ausnahmevorschrift des $\S 4$ a Satz 1 Nr. 3 AMG für den Fall der Herstellung einer Gewebezubereitung unter der unmittelbaren fachlichen Verantwortung des herstellenden Arztes zur ausschließlichen Anwendung am eigenen Patienten nicht gelten soll, hätte er dies entsprechend geregelt und aus rechtsstaatlichen Gründen regeln müssen.

Hinsichtlich der Anwendung des Arzneimittels fordert § 4a Satz 1 Nr. 3 AMG, dass der das Arzneimittel herstellende Arzt das Arzneimittel anwendet.

Wer Anwender im Sinne des $\S$ 4a Satz 1 Nr. 3 AMG ist, wird im AMG nicht definiert. Insbesondere ist unklar, ob der herstellende Arzt auch im Rahmen der Anwendung Hilfspersonen einsetzen darf. Ausgangspunkt der Überlegung, wer Anwender im Sinne des $\S 4$ a Satz 1 Nr. 3 AMG ist, muss die in dieser Vorschrift und verfassungsrechtlich in Artikel 12 GG verankerte ärztliche Therapiefreiheit sein. Ein Arzt ist daher auch dann Anwender eines Arzneimittels, wenn er bei der Behandlung eines Patienten weisungsgebundene Hilfspersonen oder den Patienten einsetzt und sich so bei der Anwendung helfen lässt [6]. Entscheidend ist allein, dass der Arzt auch für solche Hilfstätigkeiten anderer die ausschließliche therapeutische Verantwortung trägt und jederzeit eingreifen kann. Das ist aber nur dann der Fall, wenn er das Arzneimittel in seinem unmittelbaren Einwirkungsbereich - sei es in seinen Praxisräumen, sei es beim Hausbesuch, sei es an der Unfallstelle oder bei ähnlicher Gelegenheit - unter seiner Aufsicht oder unmittelbaren Aufsichtsmöglichkeit durch weisungsgebundene Hilfskräfte oder durch den Patienten anwenden lässt [7]. Im Übrigen wird dies bestätigt durch das oben zitierte Urteil des BVerfG vom 18. März 2004.

Damit kann im Ergebnis festgehalten werden, dass die erlaubnisfreie ärztliche Eigenherstellung einer Gewebezubereitung im Sinne des $§ 4$ Abs. 30 AMG zur Anwendung am eigenen Patienten nach $\S 4$ a Satz 1 Nr. 3 AMG unter der unmittelbaren fachlichen Verantwortung des herstellenden und anwendenden Arztes auch unter Einschaltung von weisungsgebundenen Hilfspersonen rechtlich zulässig ist. Der Arzt unterliegt bei diesem Vorgang nicht dem Anwendungsbereich des AMG. Eine arzneimittelrechtliche Erlaubnis oder Genehmigung ist nicht erforderlich.

\section{Genehmigungspflicht für Gewebezubereitungen nach § 21a AMG}

Unterstellt, das AMG findet - mangels Vorliegens der Voraussetzungen der ärztlichen Eigenherstellung einer Gewebezubereitung nach $\S 4$ a Satz 1 Nr. 3 AMG - Anwendung, dann 
ist für die Gewinnung des Gewebes eine Erlaubnis nach § 20b AMG erforderlich. Ferner bedarf eine Einrichtung, die Gewebe oder Gewebezubereitungen be- oder verarbeiten, konservieren, lagern oder in den Verkehr bringen will, dann einer Erlaubnis nach § 20c AMG, wenn die sonstigen Voraussetzungen nach § 20c AMG (nichtindustrielles Verfahren, in der Europäischen Union hinreichend bekannt) gegeben sind.

Die Erlaubnis nach § 20b AMG wird der Entnahmeeinrichtung gemäß $\S 20 \mathrm{~b}$ Abs. 1 Satz 5 AMG für eine bestimmte Betriebsstätte erteilt. Entsprechendes gilt für die Erlaubnis nach $\S 20$ c AMG, wie sich $\S 20$ c Abs. 4 Satz 3 AMG entnehmen lässt. Sie wird also z.B. nur dem Krankenhaus als Erlaubnisinhaber für die Abteilung für Orthopädie erteilt. Dies entspricht der Erteilung einer Herstellungserlaubnis nach $\S 13$ AMG, die gleichfalls dem Hersteller nur für eine bestimmte Betriebsstätte erteilt wird.

Soll die Gewebezubereitung in den Verkehr gebracht werden, ist schließlich eine Genehmigung durch das Paul-EhrlichInstitut als zuständige Bundesoberbehörde erforderlich (vergleiche $\S \S 21 \mathrm{a}$ Abs. 1 Satz 1, 77 Abs. 2 AMG). Dabei stellt die «Abgabe an andere» einen Unterfall des Inverkehrbringens im Sinne des $\S 4$ Abs. 17 AMG dar.

Diesbezüglich hat von Auer [1] bereits zutreffend festgestellt, dass die Verfügungsgewalt unverändert noch gegeben sein kann - also keine «Abgabe an andere» vorliegt -, wenn der Leiter einer Klinikabteilung unmittelbar für die Herstellung der Gewebezubereitung in seiner Abteilung zuständig ist und sie auch in seiner Abteilung anwendet, wenn auch unter $\mathrm{Zu}$ hilfenahme seiner ihm unterstellten Ärzte. Bleibt die Gewebezubereitung in der Betriebsstätte, in der sie hergestellt worden ist, und wird sie in dieser Betriebsstätte angewendet, bedarf es mangels Inverkehrbringens keiner Genehmigung gemäß $§ 21 \mathrm{a}$ AMG.

Dem Vernehmen nach ist zumindest ein Teil der Landesbehörden (z.B. Nordrhein-Westfalen) der Auffassung, dass ein Inverkehrbringen allerdings dann vorliegt, wenn die Gewebezubereitung außerhalb der Räumlichkeiten der herstellenden Abteilung (also der Betriebsstätte, die Gegenstand der Erlaubnis nach § 20c AMG ist) in einer anderen Abteilung desselben Krankenhauses angewendet wird. Dann gehe die Verfügungsberechtigung in der Regel auf diejenige Abteilung über, die die Gewebezubereitung anwendet, mit der Folge, dass dafür eine Genehmigung zu beantragen ist.

Nach der weitergehenden Auffassung der Autoren ist nicht von vornherein ausgeschlossen, dass die Verfügungsgewalt auch bei der letztgenannten Konstellation unverändert fortbesteht mit der Folge, dass es mangels Inverkehrbringens keiner Genehmigung gemäß $§ 21 \mathrm{a}$ AMG bedarf. Es muss vielmehr aus den nachstehend genannten Gründen entscheidend auf die innerbetriebliche Organisation durch den Inhaber der Erlaubnis nach § 20c AMG, also z.B. des Krankenhausträgers, abgestellt werden:

Nach dem allgemeinen arzneimittelrechtlichen Sprachgebrauch wird als Abgabe im Allgemeinen die «Einräumung der
Verfügungsgewalt» an einen anderen durch körperliche Überlassung des Arzneimittels verstanden, also ein «Wechsel in der Verfügungsgewalt» [8]. Dabei ist allerdings unklar, was unter Verfügungsgewalt zu verstehen ist, insbesondere ob es hierbei auf die tatsächliche Möglichkeit und/oder die rechtliche Befugnis zu verfügen ankommt.

Nach Auffassung der Autoren ist zumindest auch auf die rechtliche Verfügungsbefugnis abzustellen. Nur dann, wenn ein anderer dazu berechtigt wird, über das Arzneimittel zu verfügen, liegt eine Abgabe an andere und damit ein Inverkehrbringen vor [9]. Entscheidend ist daher, ob die Verfügungsgewalt im Sinne der Verfügungsberechtigung wechselt [10].

Der in der Literatur vertretenen Auffassung, wonach für eine arzneimittelrechtlich relevante Abgabe die Besitzeinräumung im Sinne einer Übertragung der tatsächlichen Verfügungsgewalt ausreichend sein soll [11], kann daher nicht zugestimmt werden. Wenn es allein auf die tatsächlichen Besitzverhältnisse ankäme, müsste nämlich konsequenterweise auch die (auftragsgemäße) Übergabe eines Arzneimittels an einen bloBen Boten (z.B. Taxifahrer), der ein Arzneimittel ausfährt und dem bestimmungsgemäßen Empfänger aushändigt, eine Abgabe an andere und damit ein Inverkehrbringen darstellen. Dass dies nicht zutreffend sein kann, liegt auf der Hand. Der Bote wird nämlich von seinem Auftraggeber gerade nicht berechtigt, über das Arzneimittel (rechtlich) zu verfügen, sondern lediglich dazu, es - zum Zwecke der Übermittlung - zu besitzen und körperlich auszuhändigen. Auch die von Kloesel angeführte Begründung [12], wonach ein «Bote, der Arzneimittel ausfährt, ... in der Regel nicht Täter einer verbotswidrigen Abgabe [ist], weil er nicht verfügungsberechtigt ist», lässt erkennen, dass für die Verfügungsberechtigung also immer auch das rechtliche Dürfen, und nicht nur das tatsächliche Können für die Frage der Abgabe eine entscheidende Rolle spielt. Die rechtliche Komponente kann daher bei der Verfügungsgewalt nicht gänzlich unberücksichtigt bleiben. Auf diese Weise durchdringt das Zivilrecht das dem Öffentlichen Recht zuzuordnende Arzneimittelrecht. Eine gesicherte zivilrechtliche Terminologie kann nicht auf dem Altar vermeintlicher arzneimittelrechtlicher Erfordernisse geopfert werden. Dies wäre nur durch eine ausdrückliche gesetzgeberische Entscheidung möglich. Somit kann der Auffassung von Kloesel zwar im Ergebnis, nicht aber mit der Begründung zugestimmt werden.

Kommt es auch auf die rechtliche Verfügungsbefugnis an, dann ist der Träger des Krankenhauses, also z.B. die juristische Person GmbH, Inhaber der Verfügungsgewalt bei der Herstellung des Arzneimittels und behält dieser die Verfügungsgewalt unverändert auch bei der Anwendung des Arzneimittels durch einen Arzt innerhalb des rechtlichen und örtlichen tatsächlichen Zuständigkeitsbereichs der juristischen Person. Daher liegt rechtlich kein Wechsel in der Verfügungsgewalt vor, wenn in einer Abteilung des Krankenhauses die Gewebezubereitung hergestellt und dieses Arzneimittel in einer ande- 
ren Abteilung desselben Krankenhausträgers, nämlich dem alleinigen Inhaber der Verfügungsgewalt im Sinne der Verfügungsberechtigung, angewendet wird [13]. Der Arzt übt die tatsächliche Sachherrschaft lediglich für das Krankenhaus als Erlaubnisinhaber aus, das ihm gegenüber durch vorgesetzte Ärzte oder die Geschäftsführung arbeitsrechtlich (oder dienstrechtlich) unverändert weiterhin weisungsbefugt bleibt (vergleiche Besitzdiener im Sinne des. § 855 Bürgerliches Gesetzbuch). Eine Abgabe an andere, also an andere Dritte, liegt in diesem Fall gerade nicht vor. Daher ist es im Ergebnis eine Frage der innerbetrieblichen Organisation durch den Krankenhausträger und ist es dessen Aufgabe, insoweit die organisatorischen und rechtlichen Voraussetzungen zu schaffen, worauf von Auer [1] zutreffend hingewiesen hat. Wird die Gewebezubereitung z.B. durch den Leiter einer Krankenhausabteilung hergestellt und einer anderen Abteilung überlassen, ist dabei Sorge zu tragen, dass der unmittelbare Einwirkungsbereich auch bei der Anwendung der Gewebezubereitung fortbesteht. Dies dürfte zugleich mit einer - vertraglich begründeten - Einschränkung der Therapiefreiheit der betroffenen Ärzte verbunden sein.

Dem steht auch nicht das oben zitierte Urteil des BVerfG entgegen. Das BVerfG hat zwar festgestellt, dass die Weitergabe an andere Ärzte ein Inverkehrbringen sei, jedoch hat das Gericht die Abgabe an einen anderen Arzt zur eigenverantwortlichen Anwendung an seinem Patienten gemeint, wie der Zusammenhang ergibt [14].

Allerdings wird in der Literatur im Zusammenhang mit der Frage des Erfordernisses einer Herstellungserlaubnis nach $\S 13$ AMG und der dort gleichfalls relevanten Frage, ob eine Abgabe im Sinne des $§ 13$ Abs. 1 Satz 3 AMG vorliegt, die Auffassung vertreten, dass «keine Identität besteht zwischen der herstellenden und der anwendenden Person, wenn die Anwendung in unterschiedlichen Abteilungen eines Krankenhauses erfolgt, z.B. eine Abteilung Blut gewinnt und aufbereitet und die andere Abteilung das Produkt Patienten verabreicht» [15]. § 13 Abs. 1 Satz 3 AMG lautet: «Eine Abgabe an andere im Sinne des Satzes 1 liegt vor, wenn die Person, die das Arzneimittel herstellt, eine andere ist als die, die es anwendet.»

Diese Auffassung kann, wenn überhaupt für ein Krankenhaus, nur dann gelten, wenn dem - anderen - nämlich therapierenden Arzt die originäre Verantwortung für die Anwendung des Arzneimittels übertragen wird. Bei einem Fortbestehen der Verantwortung des herstellenden Arztes auch für die Therapie gilt das jedenfalls nicht.

Im Übrigen ist aber zu beachten, dass $\S 13$ Abs. 1 Satz 3 AMG für den erörterten Fall der Gewebe und Gewebezubereitungen ohnehin nicht gelten dürfte: $§ 13$ Abs. 1 Satz 4 AMG schließt nämlich die Anwendbarkeit des § 13 Abs. 1 Satz 1 AMG insoweit ausdrücklich aus; § 13 Abs. 1 Satz 3 AMG wiederum bezieht sich auf den - in diesem Fall gerade nicht anwendbaren - Satz 1. Die Definition im Satz 3 ist folglich auf Gewebe und Gewebezubereitungen nicht anzuwenden.
Ferner handelt es sich bei $\S 13$ Abs. 1 Satz 3 AMG um eine Legaldefinition für den speziellen Bereich der Herstellung. Die Begriffsbestimmung gilt - mangels Verweises - nicht zwingend für das gesamte $\mathrm{AMG}$, insbesondere nicht auch für den zulassungsrelevanten Begriff des Inverkehrbringens im Sinne des $\S 4$ Abs. 17 AMG. Es ist daher davon abzuraten, die in $\S 13$ Abs. 1 Satz 3 AMG enthaltene herstellungsrechtliche Definition den sonstigen Vorschriften des AMG, insbesondere solchen, die das zulassungsrelevante Inverkehrbringen betreffen, «überzustülpen».

Die Richtigkeit dieser Auffassung findet im Übrigen ihre Bestätigung durch die insoweit gegebenen und unbestrittenen rechtlichen Verhältnisse bei der Lohnherstellung (§ 9 AMWHV [16]). Es besteht nämlich im Ergebnis kein Zweifel daran, dass derjenige, der gewerblich ein Arzneimittel herstellt, um es - in Erfüllung seiner vertraglichen Verpflichtung - ausschließlich seinem Auftraggeber zum Inverkehrbringen zu überlassen, das Fertigarzneimittel im Sinne des $\S 4$ Abs. 1 Satz 1 AMG nicht im Sinne des $\$ 4$ Abs. 17 AMG in den Verkehr bringt. Eine «Abgabe an andere» liegt in diesem Falle nicht vor. Ansonsten dürfte der Lohnauftragnehmer das Arzneimittel nur dann dem Lohnauftraggeber überlassen, wenn der Lohnauftragnehmer für das von ihm hergestellte Arzneimittel über eine Zulassung nach § 21 AMG oder Genehmigung nach § 21a AMG verfügt. Gerade im Falle der Lohnherstellung ist aber meist der Lohnauftraggeber, niemals aber der Lohnauftragnehmer, Inhaber der Zulassung bzw. Genehmigung für das Inverkehrbringen des hergestellten Arzneimittels [17].

Hinzu kommt folgende Überlegung. Der Inhaber einer Zulassung oder Genehmigung darf sein Arzneimittel in den Verkehr bringen. Die Zulassung oder Genehmigung ist jedoch keinesfalls betriebsstättenbezogen wie die Herstellungserlaubnisse gemäß $\S 13,20$ b und 20c AMG. Wo auch immer der Berechtigte seinen Sitz oder seine Betriebsstätten hat von dort darf er das Arzneimittel in den Verkehr bringen. Das Versenden des zugelassenen Arzneimittels von einer Betriebsstätte an eine andere des Berechtigten ist unbestrittenermaßen noch kein Inverkehrbringen. Nichts anderes kann gelten, wenn ein Arzneimittel im Verfügungsbereich des Erlaubnisinhabers verbleibt und diesen überhaupt nicht verlässt.

Für ein solches Verständnis spricht auch eine neue Definition des EuGH [18]. Der EuGH sieht ein Produkt erst dann als in den Verkehr gebracht an, wenn es den vom Hersteller eingerichteten Prozess der Herstellung verlassen hat und in einen Prozess der Vermarktung eingetreten ist, in dem es in ge- oder verbrauchsfertigem Zustand öffentlich angeboten wird.

Auch wenn das Urteil sich auf den Bereich des Produkthaftungsgesetzes bezieht, kann dieser Entscheidung doch entnommen werden, dass für die Frage des Inverkehrbringens im Allgemeinen entscheidend ist, welchem Personenkreis ein Produkt zur Verfügung gestellt wird. Wird es einem prinzipiell unbeschränkten Empfängerkreis - gegebenenfalls unter Ein- 
haltung eines vorgeschriebenen Vertriebswegs - angeboten, liegt ein Inverkehrbringen vor; ansonsten eben nicht.

Eine Gewebezubereitung, die in einer Krankenhausabteilung ausschließlich hergestellt wird, um sie im Bedarfsfalle einer anderen Person in einer anderen Abteilung zur Anwendung $\mathrm{zu}$ überlassen, wird gerade nicht öffentlich im Sinne der Rechtsprechung des EuGH angeboten. Vielmehr verlässt die Gewebezubereitung nicht die Räumlichkeiten des Krankenhauses und somit den rechtlichen Herrschaftsbereich des Erlaubnisinhabers, nämlich des Krankenhauses. Es tritt keine Änderung der Verfügungsbefugnis ein. In diesem Falle ist daher eine Abgabe an andere als Unterfall des Inverkehrbringens zu verneinen.

Interessanterweise findet sich im US-amerikanischen Code of Federal Regulations [19] für den Bereich der Medizinprodukte eine Legaldefinition des Begriffs «Commercial distribution», die einen ähnlichen Inhalt hat und wie folgt lautet:

«Commercial distribution means any distribution of a device intended for human use which is held or offered for sale but does not include the following:

Internal or interplant transfer of a device between establishments within the same parent, subsidiary, and/or affiliate company.»

Ferner wird dem Sinn und Zweck des AMG, nämlich der Gewährleistung der Arzneimittelsicherheit und dem Interesse an einer ordnungsgemäßen Arzneimittelversorgung (vergleiche $\S 1$ AMG), im Bereich der Gewebe und Gewebezubereitungen nunmehr hinreichend durch das Erfordernis einer Erlaubnis nach § 20b AMG für die Gewinnung von Gewebe und nach § 20c AMG für die Be- oder Verarbeitung, Konservierung, Lagerung oder das Inverkehrbringen Rechnung getragen. Eine darüber hinaus gehende Gefährdung, die durch das Erfordernis einer generellen Genehmigung der Gewebezubereitung nach § 21a AMG abgesichert werden müsste, wird im Falle einer innerhalb des Herrschaftsbereichs desselben Krankenhausträgers hergestellten und angewendeten Gewebezubereitung nicht geschaffen. Auf der Grundlage des § 4a Satz 1 Nr. 3 AMG dürfte derselbe Arzt zweifelsfrei, wenn er das Arzneimitteln in einer Abteilung herstellt, dieses Arzneimittel in einer anderen Abteilung am Patienten anwenden, wenn dies unter seiner unmittelbaren fachlichen Verantwortung in dem dargestellten Sinne geschieht. Er unterläge in diesem Fall dem AMG überhaupt nicht. Da in dem erörterten Fall jedenfalls eine staatliche Kontrolle mit Erlaubnisvorbehalt ( $\$ 20 \mathrm{~b}$, 20c AMG) besteht und damit eine staatliche Überprüfung zur Qualitätsgewährleistung stattfindet, wäre eine noch darüber hinausgehende zusätzliche Genehmigungspflicht gemäß § 21a AMG eine Verletzung des Verhältnismäßigkeitsgrundsatzes (Artikel 20 Abs. 3 in Verbindung mit Artikel 19 Abs. 3 GG). Eine Verletzung des Gleichheitsgrundsatzes (Artikel 3 Abs. 1 GG) dürfte möglicherweise auch vorliegen, obwohl aus Artikel 3 GG keine notwendige Gleichbehandlung natürlicher und juristischer Personen folgt. Sowohl der Arzt als auch ein Krankenhaus können sich auf die Berufs- und Gewerbefreiheit des Artikels 12 GG (in Verbindung mit Artikel 19 Abs. 3 GG) berufen.

Eine generelle Genehmigungspflicht derartiger Gewebezubereitungen wird auch nicht von der Richtlinie 2004/23/EG (Geweberichtlinie) [20] gefordert, so dass auch eine richtlinienkonforme Auslegung zu keinem anderen Ergebnis führt. Nach dem 4. Erwägungsgrund dieser Richtlinie besteht ein dringender Bedarf an einheitlichen Rahmenbedingungen für die Gewährleistung hoher Qualitäts- und Sicherheitsstandards bei der Beschaffung, Testung, Verarbeitung, Lagerung und Verteilung von Geweben und Zellen in der Gemeinschaft. Dabei bezeichnet der Ausdruck «Verteilung» die Beförderung und Abgabe von zur Verwendung beim Menschen bestimmten Geweben oder Zellen (Artikel 3 Buchstabe k Richtlinie 2004/23/EG). Eine Vorschrift, die besagt, dass eine Verteilung auch dann vorliegt, wenn die in einer Abteilung eines Krankenhauses, also einer Gewebeeinrichtung im Sinne des Artikels 3 Buchstabe o Richtlinie 2004/23/EG, hergestellte Gewebezubereitung in einer anderen Abteilung desselben Krankenhauses angewendet wird, gibt es nicht. Dass eine Verteilung in diesem Sinne auch dann gegeben sein soll, wenn die Räumlichkeiten des Krankenhausträgers nicht verlassen werden und der unmittelbare Einwirkungsbereich des Trägers einschränkungslos erhalten bleibt, lässt sich der Richtlinie nicht entnehmen. Die Richtlinie beschäftigt sich mit dieser Konstellation nicht, sondern zielt vielmehr darauf ab, dass die Gewebeeinrichtung Gewebe und Zellen verteilt, also an einen anderen Ort befördert und abgibt. Dabei spricht schon der in der Definition genannte Begriff «Beförderung» dafür, dass eine gewisse Distanz zu überbrücken ist, was bei einem Verbringen von einer Abteilung zu einer anderen eher nicht der Fall sein dürfte. Auch die Richtlinie 2006/86/EG [21], die in ihrem Anhang II (Anforderungen für die Zulassung von Verarbeitungsverfahren für Gewebe und Zellen im Sinne des Artikels 4) unter Buchstabe D (Verteilung und Rückruf) Anforderungen hinsichtlich der Verteilung regelt, lässt erkennen, dass eher von einem Verlassen der Räumlichkeiten des Krankenhausträgers ausgegangen wird, und nicht von einem lediglich innerbetrieblichen Transport [22].

Im Ergebnis kann festgehalten werden, dass keine Genehmigung von Gewebezubereitungen nach § 21a AMG erforderlich ist, wenn die in einem Krankenhaus hergestellte Gewebezubereitung innerhalb desselben Krankenhauses an einen anderen als den herstellenden Arzt zur Anwendung an seinem Patienten abgegeben wird, sofern durch die innerbetriebliche Organisation der unmittelbare rechtliche Einwirkungsbereich des Krankenhausträgers fortbesteht. Es liegt dann keine «Abgabe an andere» und damit kein Inverkehrbringen im Sinne des $§ 4$ Abs. 17 AMG vor. 


\section{Literatur}

1 Von Auer F: Das Gewebegesetz - Hintergründe und Konsequenzen. Transfus Med Hemother 2008; 35(6):407-413.

2 Gesetz über Qualität und Sicherheit von menschlichen Geweben und Zellen (Gewebegesetz) vom 20. Juli 2007 (BGBl. I S. 1574).

3 Gesetz über den Verkehr mit Arzneimitteln in der Fassung der Bekanntmachung vom 12. Dezember 2005 (BGBl. I S. 3394), zuletzt geändert durch Gesetz vom 23. November 2007 (BGBl. I S. 2631).

4 so Sander, Kommentar zum Arzneimittelrecht, Stand: August 2006, Band 1, § 4a Erl. 4, unter Hinweis auf die außer Kraft getretenen Vorschriften des $\S \S 5$ Abs. 3 und 6 Abs. 2 PharmBetrV.

5 Urteil des BVerwG vom 18. März 2004, Az.: 3 C 16/03, abgedruckt in: NVwZ 2005, S. $87 \mathrm{f}$

6 vgl. zu § 13 AMG Urteil des Bayerischen Verwaltungsgerichtshofes vom 27. März 1997, Az.: 25 B 96 2040, abgedruckt in: Pharma Recht 1997, S. 481; Urteil des BVerwG, a.a.O.

7 vgl. Urteil des Bayerischen Verwaltungsgerichtshofes, a.a.O.

$8 \mathrm{Kloesel} / \mathrm{Cyran} /$ Feiden/Pabel, Arzneimittelrecht, Kommentar, 3. Auflage, Stand: März 2007, § 4 Anm. 57; zu § 13 AMG Sander, Kommentar zum Arzneimittelrecht, Stand: August 2006, Band 2, 13 AMG Erl. 4.

9 i.d.S. auch Hasskarl/Ostertag, Hämatopoetische Stammzellen aus Nabelschnurblut - Medizinische und rechtliche Aspekte, NJW 2002, S. 1773 f.
10 Auch nach von Auer ist der Begriff des Inverkehrbringens erfüllt ist, wenn die «Verfügungsgewalt im Sinne der Verfügungsberechtigung» wechselt, siehe [1].

11 Kloesel/Cyran/Feiden/Pabel, a.a.O., § 4 Anm. 57.

$12 \mathrm{Kloesel} / \mathrm{Cyran} /$ Feiden/Pabel, a.a.O., unter Hinweis auf RGSt 14,35, 36; BGH LRE 1, 82.

13 In diese Richtung geht auch die bereits 1993 vertretene Auffassung im Hinblick auf $\S 13$ AMG von Wolfslast/Rosenau, «Zur Anwendung des Arzneimittelgesetzes auf die Entnahme von Organ- und Gewebetransplantaten», in: Neue Juristische Wochenschrift, 1993, S. 2348 (2350)

14 Entsprechendes gilt im übrigen im Hinblick auf den Beschluss des OVG NW vom 2. August 1994, Az.: 13 B 584/94. Auch das OVG hat i.R.d. § 13 AMG darauf abgestellt, ob der Hersteller die Verfügungsgewalt insofern verliert, als die sie erlangenden Ärzte allein medizinisch-fachlich entscheiden können, ob und wie die Arzneimittel zur Anwendung kommen.

15 Kloesel/Cyran/Feiden/Pabel, a.a.O., § 13 Anm. 30b; so auch Sander, a.a.O., § 13 Erl. 4.

16 Verordnung über die Anwendung der Guten Herstellungspraxis bei der Herstellung von Arzneimitteln und Wirkstoffen und über die Anwendung der Guten fachlichen Praxis bei der Herstellung von Produkten menschlicher Herkunft (Arzneimittelund Wirkstoffherstellungsverordnung - AMWHV) vom 3. November 2006 (BGBl. I S. 2523), zuletzt geändert durch Verordnung vom 26. März 2008 (BGBl. I S. 521) einschließlich der bekannt gemachten EG-Leitlinien für die Gute Herstellungspraxis (Bundesanzeiger Nr. 210 vom 9. November 2006, S. 6887)
17 Dies bleibt bei Sander, a.a.O., § 4 Erl. 21 unberücksichtigt.

18 Urteil des EuGH vom 9. Februar 2006 - C-127/04 (Declan O'Byrne/Sanofi Pasteur MSD Ltd. unter anderem), abgedruckt in: NJW 2006, $825 \mathrm{f}$.

19 Title 21 CFR $\$ 807,3$ (b) (1).

20 Richtlinie 2004/23/EG des Europäischen Parlaments und des Rates vom 31. März 2004 zur Festlegung von Qualitäts- und Sicherheitsstandards für die Spende, Beschaffung, Testung, Verarbeitung, Konservierung, Lagerung und Verteilung von menschlichen Geweben und Zellen (ABl. EU Nr. L 102/48 vom 7. April 2004)

21 Richtlinie 2006/86/EG der Kommission vom 24 Oktober 2006 zur Umsetzung der Richtlinie 2004/23/EG des Europäischen Parlaments und des Rates hinsichtlich der Anforderungen an die Rückverfolgbarkeit, der Meldung schwerwiegender Zwischenfälle und unerwünschter Reaktionen sowie bestimmter technischer Anforderungen an die Kodierung, Verarbeitung, Konservierung, Lagerung und Verteilung von menschlichen Geweben und Zellen (ABl. EU Nr. L 294/32 vom 25. Oktober 2006).

22 siehe z.B. «kritische Transportbedingungen» (Richtlinie 2006/86/EG, Anhang II, Buchstabe D Ziffer 1).

23 Bundesärtekammer Kassenärztliche Bundesvereinigung: Bekanntmachungen - Persönliche Leistungserbringung: Möglichkeiten und Grenzen der Delegation ärztlicher Leistungen. Stand: 29. August 2008. Dtsch Ärztebl 2008;105(41):A2173-A2177. 